\title{
Toward Actinide Molecular Magnetic Materials: Coordination Polymers of U(IV) and the Organic Acceptors TCNQ and TCNE
}

\author{
Eric J. Schelter, ${ }^{*}$ David E. Morris, Brian L. Scott, Joe D. Thompson, \\ and Jaqueline L. Kiplinger ${ }^{*}$ \\ Los Alamos National Laboratory, Los Alamos, NM 87545 \\ Fax: +015056679905; Tel: +01505665 9553; \\ E-mail: schelter@lanl.gov
}

Supporting Information:

Figure S1 - Electrochemical Response for [K-18-crown-6][TCNQ] (3) and [K-18-crown$6][\mathrm{TCNE}]$ (4) Collected in $\sim 0.1 \mathrm{M}\left[\mathrm{Bu}_{4} \mathrm{~N}\right]\left[\mathrm{B}\left(\mathrm{C}_{6} \mathrm{~F}_{5}\right)_{4}\right] / \mathrm{THF}$ at Pt Working Electrode. (S2)

Figure S2 - Magnetic Susceptibility Data for [K-18-crown-6][TCNE] (4) Collected from 2-350 K. (S3)

Table S1 - Crystallographic Details for [K-18-crown-6][TCNE] $\mathrm{CH}_{3} \mathrm{CN},(3) \cdot \mathrm{CH}_{3} \mathrm{CN}$. (S4)

Table S2 - Table of Bond Distances and Angles for [K-18-crown-6][TCNE] $\mathrm{CH}_{3} \mathrm{CN}$, (3) $\mathrm{CH}_{3} \mathrm{CN}$. (S5-S7). 
Figure S1 - Electrochemical Response for [K-18-crown-6][TCNQ] (3) and [K-18-crown6][TCNE] (4) Collected in $\sim 0.1 \mathrm{M}\left[\mathrm{Bu}_{4} \mathrm{~N}\right]\left[\mathrm{B}\left(\mathrm{C}_{6} \mathrm{~F}_{5}\right)_{4}\right] /$ THF at Pt Working Electrode.
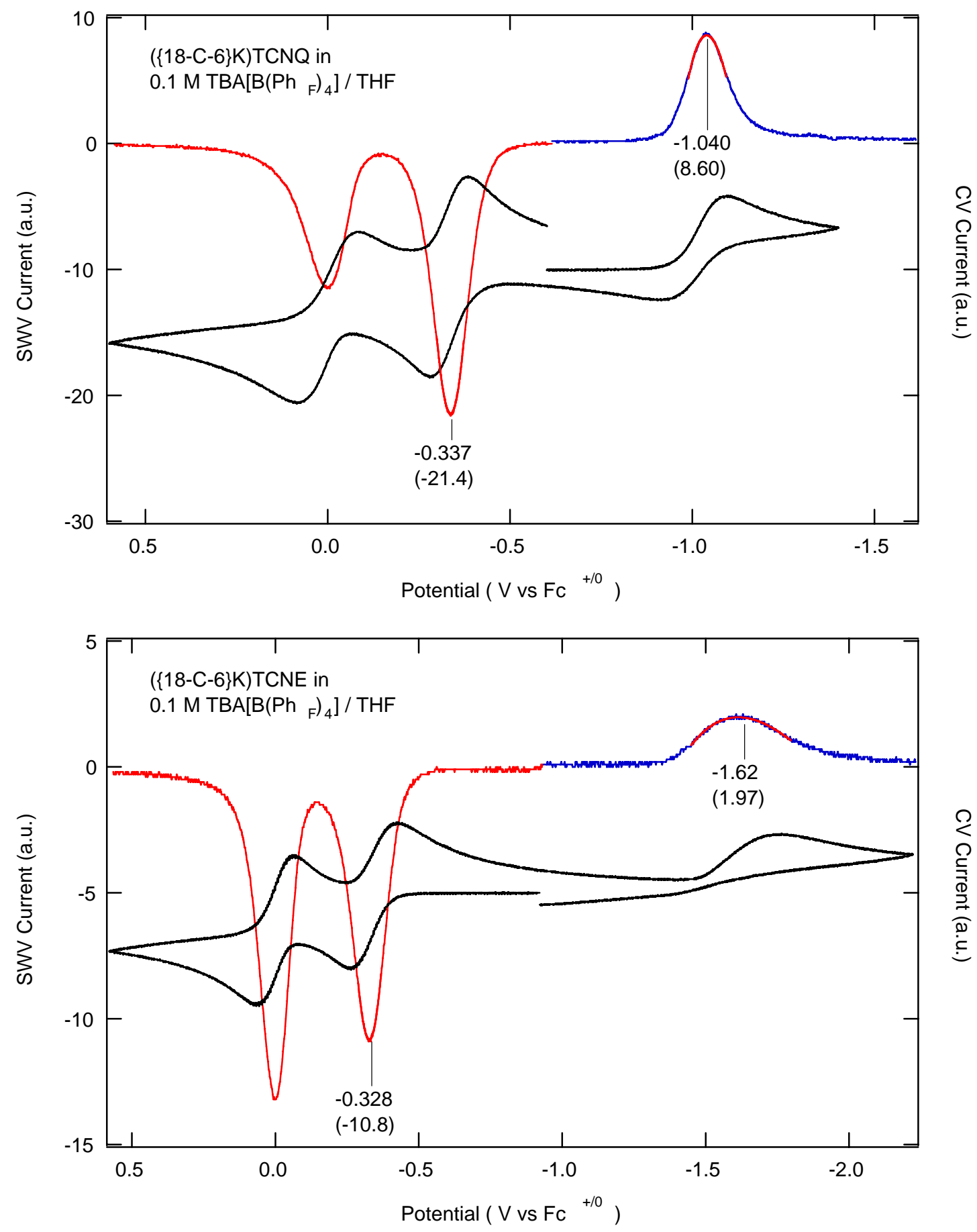
Figure S2 - Magnetic Susceptibility Data for [K-18-crown-6][TCNE] (4) Collected from 2-350 $\mathrm{K}$.

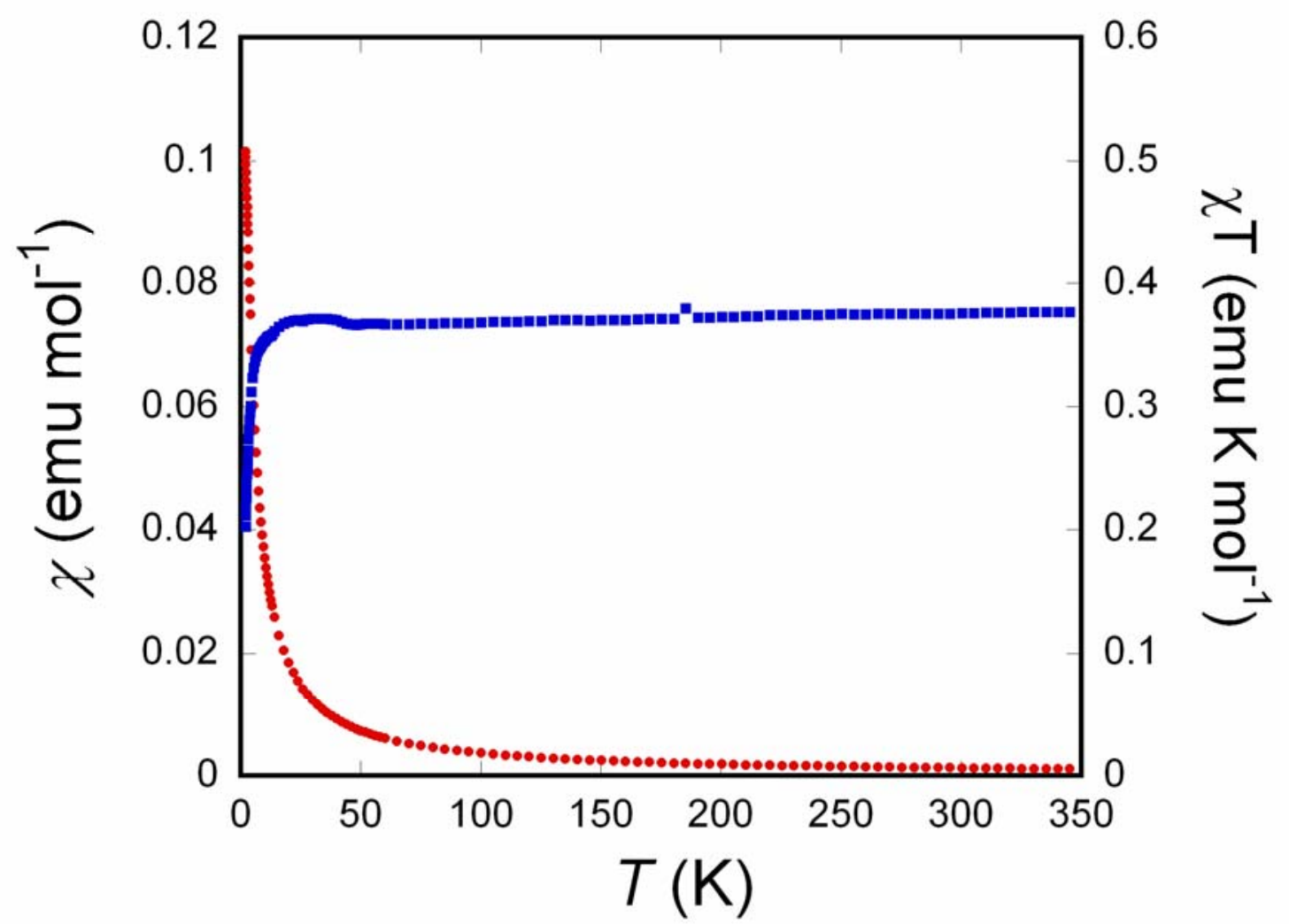


Table S1 - Crystallographic Details for [K-18-crown-6][TCNE] $\mathrm{CH}_{3} \mathrm{CN},(3) \mathrm{CH}_{3} \mathrm{CN}$.

\begin{tabular}{|c|c|c|}
\hline Empirical formula & \multicolumn{2}{|l|}{$\mathrm{C}_{20} \mathrm{H}_{27} \mathrm{~K} \mathrm{~N}_{5} \mathrm{O}$} \\
\hline Formula weight & \multicolumn{2}{|l|}{392.57} \\
\hline Temperature & \multicolumn{2}{|l|}{ 293(2) K } \\
\hline Wavelength & \multicolumn{2}{|l|}{$0.71073 \AA$} \\
\hline Crystal system & \multicolumn{2}{|l|}{ monoclinic } \\
\hline Space group & \multicolumn{2}{|l|}{$\mathrm{C} 2 / \mathrm{c}$} \\
\hline \multirow[t]{3}{*}{ Unit cell dimensions } & $\mathrm{a}=27.054(3) \AA$ & $\alpha=90^{\circ}$ \\
\hline & $\mathrm{b}=12.1104(15) \AA$ & $\beta=98.159(2)^{\circ}$ \\
\hline & $\mathrm{c}=15.1194(19) \AA$ & $\gamma=90^{\circ}$ \\
\hline Volume & \multicolumn{2}{|l|}{$4903.6(11) \AA^{3}$} \\
\hline $\mathrm{Z}$ & \multicolumn{2}{|l|}{8} \\
\hline Density (calculated) & \multicolumn{2}{|l|}{$1.064 \mathrm{Mg} / \mathrm{m}^{3}$} \\
\hline Absorption coefficient & \multicolumn{2}{|l|}{$0.233 \mathrm{~mm}^{-1}$} \\
\hline $\mathrm{F}(000)$ & \multicolumn{2}{|l|}{1672} \\
\hline Crystal size & \multicolumn{2}{|c|}{$0.14 \times 0.08 \times 0.04 \mathrm{~mm}^{3}$} \\
\hline$\Theta$ Range for data collection & \multicolumn{2}{|c|}{1.52 to $25.37^{\circ}$} \\
\hline Index ranges & \multicolumn{2}{|c|}{$-32 \leq \mathrm{h} \leq 32,-14 \leq \mathrm{k} \leq 14,-18 \leq 1 \leq 18$} \\
\hline Reflections collected & \multicolumn{2}{|c|}{24302} \\
\hline Independent reflections & \multicolumn{2}{|c|}{$4499[\mathrm{R}(\mathrm{int})=0.0962]$} \\
\hline Completeness to $\Theta=25.00^{\circ}$ & \multicolumn{2}{|c|}{$100.0 \%$} \\
\hline Max. and min. transmission & \multicolumn{2}{|l|}{0.9907 and 0.9681} \\
\hline Refinement method & \multicolumn{2}{|c|}{ Full-matrix least-squares on $\mathrm{F}^{2}$} \\
\hline Data / restraints / parameters & \multicolumn{2}{|c|}{$4499 / 10 / 268$} \\
\hline Goodness-of-fit on $\mathrm{F}^{2}$ & \multicolumn{2}{|l|}{0.829} \\
\hline Final $R$ indices $[I>2 \sigma(I)]$ & \multicolumn{2}{|c|}{$\mathrm{R} 1=0.0537, \mathrm{wR} 2=0.1333$} \\
\hline $\mathrm{R}$ indices (all data) & \multicolumn{2}{|c|}{$\mathrm{R} 1=0.0975, \mathrm{wR} 2=0.1590$} \\
\hline Largest diff. peak and hole & \multicolumn{2}{|c|}{0.196 and -0.190 e. $\AA^{-3}$} \\
\hline
\end{tabular}


Table S3 - Table of Bond Distances and Angles for [K-18-crown-6][TCNE] $\mathrm{CH}_{3} \mathrm{CN}$, (3) $\mathrm{CH}_{3} \mathrm{CN}$.

\begin{tabular}{|c|c|c|c|}
\hline $\mathrm{K}(1)-\mathrm{O}(3)$ & $2.791(2)$ & $\mathrm{C}(13)-\mathrm{C}(18)$ & $1.469(9)$ \\
\hline $\mathrm{K}(1)-\mathrm{N}(2) \# 1$ & $2.796(3)$ & $C(13)-C(17)$ & $1.505(9)$ \\
\hline $\mathrm{K}(1)-\mathrm{O}(6)$ & $2.799(2)$ & $C\left(13^{\prime}\right)-C(14)$ & $1.072(8)$ \\
\hline $\mathrm{K}(1)-\mathrm{O}(5)$ & $2.799(2)$ & $C\left(13^{\prime}\right)-C(17)$ & $1.409(7)$ \\
\hline $\mathrm{K}(1)-\mathrm{O}(4)$ & $2.802(2)$ & $C\left(13^{\prime}\right)-C\left(14^{\prime}\right)$ & $1.423(8)$ \\
\hline $\mathrm{K}(1)-\mathrm{O}(2)$ & $2.824(2)$ & $C\left(13^{\prime}\right)-C(16)$ & $1.457(7)$ \\
\hline $\mathrm{K}(1)-\mathrm{N}(1)$ & $2.838(3)$ & $\mathrm{C}(14)-\mathrm{C}\left(14^{\prime}\right)$ & $0.985(8)$ \\
\hline $\mathrm{K}(1)-\mathrm{O}(1)$ & $2.839(2)$ & $C(14)-C(15)$ & $1.432(8)$ \\
\hline $\mathrm{O}(1)-\mathrm{C}(12)$ & $1.417(4)$ & $C(14)-C(16)$ & $1.490(8)$ \\
\hline $\mathrm{O}(1)-\mathrm{C}(1)$ & $1.427(4)$ & $C\left(14^{\prime}\right)-C(18)$ & $1.455(8)$ \\
\hline $\mathrm{O}(2)-\mathrm{C}(3)$ & $1.422(3)$ & $C\left(14^{\prime}\right)-C(15)$ & $1.483(8)$ \\
\hline $\mathrm{O}(2)-\mathrm{C}(2)$ & $1.426(3)$ & & \\
\hline $\mathrm{O}(3)-\mathrm{C}(5)$ & $1.427(4)$ & $\mathrm{O}(3)-\mathrm{K}(1)-\mathrm{N}(2) \# 1$ & $85.59(8)$ \\
\hline $\mathrm{O}(3)-\mathrm{C}(4)$ & $1.428(4)$ & $\mathrm{O}(3)-\mathrm{K}(1)-\mathrm{O}(6)$ & $175.55(7)$ \\
\hline $\mathrm{O}(4)-\mathrm{C}(7)$ & $1.426(4)$ & $\mathrm{N}(2) \# 1-\mathrm{K}(1)-\mathrm{O}(6)$ & $89.96(8)$ \\
\hline $\mathrm{O}(4)-\mathrm{C}(6)$ & $1.435(4)$ & $\mathrm{O}(3)-\mathrm{K}(1)-\mathrm{O}(5)$ & $119.29(7)$ \\
\hline $\mathrm{O}(5)-\mathrm{C}(9)$ & $1.423(4)$ & $\mathrm{N}(2) \# 1-\mathrm{K}(1)-\mathrm{O}(5)$ & $84.84(8)$ \\
\hline $\mathrm{O}(5)-\mathrm{C}(8)$ & $1.424(4)$ & $\mathrm{O}(6)-\mathrm{K}(1)-\mathrm{O}(5)$ & $60.33(6)$ \\
\hline $\mathrm{O}(6)-\mathrm{C}(11)$ & $1.413(4)$ & $\mathrm{O}(3)-\mathrm{K}(1)-\mathrm{O}(4)$ & $61.02(6)$ \\
\hline $\mathrm{O}(6)-\mathrm{C}(10)$ & $1.420(4)$ & $\mathrm{N}(2) \# 1-\mathrm{K}(1)-\mathrm{O}(4)$ & $96.86(8)$ \\
\hline $\mathrm{N}(1)-\mathrm{C}(15)$ & $1.128(4)$ & $\mathrm{O}(6)-\mathrm{K}(1)-\mathrm{O}(4)$ & $119.86(7)$ \\
\hline $\mathrm{N}(2)-\mathrm{C}(16)$ & $1.121(4)$ & $\mathrm{O}(5)-\mathrm{K}(1)-\mathrm{O}(4)$ & $60.97(7)$ \\
\hline $\mathrm{N}(2)-\mathrm{K}(1) \# 2$ & $2.796(3)$ & $\mathrm{O}(3)-\mathrm{K}(1)-\mathrm{O}(2)$ & $60.05(6)$ \\
\hline $\mathrm{N}(3)-\mathrm{C}(17)$ & $1.118(4)$ & $\mathrm{N}(2) \# 1-\mathrm{K}(1)-\mathrm{O}(2)$ & $88.53(8)$ \\
\hline $\mathrm{N}(4)-\mathrm{C}(18)$ & $1.118(4)$ & $\mathrm{O}(6)-\mathrm{K}(1)-\mathrm{O}(2)$ & $119.73(6)$ \\
\hline $\mathrm{C}(1)-\mathrm{C}(2)$ & $1.487(4)$ & $\mathrm{O}(5)-\mathrm{K}(1)-\mathrm{O}(2)$ & $173.37(7)$ \\
\hline $\mathrm{C}(3)-\mathrm{C}(4)$ & $1.485(4)$ & $\mathrm{O}(4)-\mathrm{K}(1)-\mathrm{O}(2)$ & $120.12(6)$ \\
\hline$C(5)-C(6)$ & $1.499(5)$ & $\mathrm{O}(3)-\mathrm{K}(1)-\mathrm{N}(1)$ & $92.17(8)$ \\
\hline$C(7)-C(8)$ & $1.499(5)$ & $\mathrm{N}(2) \# 1-\mathrm{K}(1)-\mathrm{N}(1)$ & $176.23(10)$ \\
\hline$C(9)-C(10)$ & $1.484(5)$ & $\mathrm{O}(6)-\mathrm{K}(1)-\mathrm{N}(1)$ & $92.26(8)$ \\
\hline $\mathrm{C}(11)-\mathrm{C}(12)$ & $1.495(5)$ & $\mathrm{O}(5)-\mathrm{K}(1)-\mathrm{N}(1)$ & $98.91(8)$ \\
\hline $\mathrm{C}(13)-\mathrm{C}\left(14^{\prime}\right)$ & $0.927(8)$ & $\mathrm{O}(4)-\mathrm{K}(1)-\mathrm{N}(1)$ & $84.67(8)$ \\
\hline $\mathrm{C}(13)-\mathrm{C}\left(13^{\prime}\right)$ & $0.992(8)$ & $\mathrm{O}(2)-\mathrm{K}(1)-\mathrm{N}(1)$ & $87.72(8)$ \\
\hline$C(13)-C(14)$ & $1.383(10)$ & $\mathrm{O}(5)-\mathrm{C}(9)-\mathrm{C}(10)$ & 108.9(3) \\
\hline
\end{tabular}




\begin{tabular}{|c|c|c|c|}
\hline $\mathrm{O}(3)-\mathrm{K}(1)-\mathrm{O}(1)$ & $118.63(6)$ & $\mathrm{O}(6)-\mathrm{C}(10)-\mathrm{C}(9)$ & 109.1(3) \\
\hline $\mathrm{N}(2) \# 1-\mathrm{K}(1)-\mathrm{O}(1)$ & $79.37(7)$ & $\mathrm{O}(6)-\mathrm{C}(11)-\mathrm{C}(12)$ & $108.6(3)$ \\
\hline $\mathrm{O}(6)-\mathrm{K}(1)-\mathrm{O}(1)$ & $60.16(6)$ & $\mathrm{O}(1)-\mathrm{C}(12)-\mathrm{C}(11)$ & $109.0(3)$ \\
\hline $\mathrm{O}(5)-\mathrm{K}(1)-\mathrm{O}(1)$ & $118.02(7)$ & $\mathrm{C}\left(14^{\prime}\right)-\mathrm{C}(13)-\mathrm{C}\left(13^{\prime}\right)$ & $95.6(9)$ \\
\hline $\mathrm{O}(4)-\mathrm{K}(1)-\mathrm{O}(1)$ & $176.22(7)$ & $C\left(14^{\prime}\right)-C(13)-C(14)$ & $45.4(6)$ \\
\hline $\mathrm{O}(2)-\mathrm{K}(1)-\mathrm{O}(1)$ & $60.41(6)$ & $C\left(13^{\prime}\right)-C(13)-C(14)$ & $50.4(5)$ \\
\hline $\mathrm{N}(1)-\mathrm{K}(1)-\mathrm{O}(1)$ & $99.11(8)$ & $\mathrm{C}\left(14^{\prime}\right)-\mathrm{C}(13)-\mathrm{C}(18)$ & $70.7(8)$ \\
\hline $\mathrm{C}(12)-\mathrm{O}(1)-\mathrm{C}(1)$ & $112.0(2)$ & $C\left(13^{\prime}\right)-C(13)-C(18)$ & $166.2(9)$ \\
\hline $\mathrm{C}(12)-\mathrm{O}(1)-\mathrm{K}(1)$ & $111.27(17)$ & $\mathrm{C}(14)-\mathrm{C}(13)-\mathrm{C}(18)$ & $116.1(8)$ \\
\hline $\mathrm{C}(1)-\mathrm{O}(1)-\mathrm{K}(1)$ & $111.67(16)$ & $C\left(14^{\prime}\right)-C(13)-C(17)$ & $160.6(9)$ \\
\hline $\mathrm{C}(3)-\mathrm{O}(2)-\mathrm{C}(2)$ & $112.3(2)$ & $C\left(13^{\prime}\right)-C(13)-C(17)$ & $65.0(7)$ \\
\hline $\mathrm{C}(3)-\mathrm{O}(2)-\mathrm{K}(1)$ & $114.12(16)$ & $\mathrm{C}(14)-\mathrm{C}(13)-\mathrm{C}(17)$ & $115.3(7)$ \\
\hline $\mathrm{C}(2)-\mathrm{O}(2)-\mathrm{K}(1)$ & $114.81(16)$ & $\mathrm{C}(18)-\mathrm{C}(13)-\mathrm{C}(17)$ & $128.6(6)$ \\
\hline $\mathrm{C}(5)-\mathrm{O}(3)-\mathrm{C}(4)$ & $112.2(2)$ & $C(13)-C\left(13^{\prime}\right)-C(14)$ & $84.1(7)$ \\
\hline $\mathrm{C}(5)-\mathrm{O}(3)-\mathrm{K}(1)$ & $113.10(18)$ & $\mathrm{C}(13)-\mathrm{C}\left(13^{\prime}\right)-\mathrm{C}(17)$ & $75.4(7)$ \\
\hline $\mathrm{C}(4)-\mathrm{O}(3)-\mathrm{K}(1)$ & $115.58(16)$ & $C(14)-C\left(13^{\prime}\right)-C(17)$ & $159.3(8)$ \\
\hline $\mathrm{C}(7)-\mathrm{O}(4)-\mathrm{C}(6)$ & $111.6(3)$ & $\mathrm{C}(13)-\mathrm{C}\left(13^{\prime}\right)-\mathrm{C}\left(14^{\prime}\right)$ & $40.4(5)$ \\
\hline $\mathrm{C}(7)-\mathrm{O}(4)-\mathrm{K}(1)$ & $113.05(19)$ & $\mathrm{C}(14)-\mathrm{C}\left(13^{\prime}\right)-\mathrm{C}\left(14^{\prime}\right)$ & $43.8(4)$ \\
\hline $\mathrm{C}(6)-\mathrm{O}(4)-\mathrm{K}(1)$ & $114.58(18)$ & $\mathrm{C}(17)-\mathrm{C}\left(13^{\prime}\right)-\mathrm{C}\left(14^{\prime}\right)$ & $115.8(6)$ \\
\hline $\mathrm{C}(9)-\mathrm{O}(5)-\mathrm{C}(8)$ & $111.6(3)$ & $C(13)-C\left(13^{\prime}\right)-C(16)$ & $154.4(8)$ \\
\hline $\mathrm{C}(9)-\mathrm{O}(5)-\mathrm{K}(1)$ & $113.82(18)$ & $\mathrm{C}(14)-\mathrm{C}\left(13^{\prime}\right)-\mathrm{C}(16)$ & $70.3(6)$ \\
\hline $\mathrm{C}(8)-\mathrm{O}(5)-\mathrm{K}(1)$ & $113.78(19)$ & $\mathrm{C}(17)-\mathrm{C}\left(13^{\prime}\right)-\mathrm{C}(16)$ & $130.2(5)$ \\
\hline $\mathrm{C}(11)-\mathrm{O}(6)-\mathrm{C}(10)$ & $112.7(2)$ & $\mathrm{C}\left(14^{\prime}\right)-\mathrm{C}\left(13^{\prime}\right)-\mathrm{C}(16)$ & $114.0(6)$ \\
\hline $\mathrm{C}(11)-\mathrm{O}(6)-\mathrm{K}(1)$ & $116.44(18)$ & $\mathrm{C}\left(14^{\prime}\right)-\mathrm{C}(14)-\mathrm{C}\left(13^{\prime}\right)$ & $87.5(7)$ \\
\hline $\mathrm{C}(10)-\mathrm{O}(6)-\mathrm{K}(1)$ & $115.17(18)$ & $C\left(14^{\prime}\right)-C(14)-C(13)$ & $42.1(5)$ \\
\hline $\mathrm{C}(15)-\mathrm{N}(1)-\mathrm{K}(1)$ & 127.1(3) & $C\left(13^{\prime}\right)-C(14)-C(13)$ & $45.5(5)$ \\
\hline $\mathrm{C}(16)-\mathrm{N}(2)-\mathrm{K}(1) \# 2$ & $132.0(3)$ & $\mathrm{C}\left(14^{\prime}\right)-\mathrm{C}(14)-\mathrm{C}(15)$ & 73.1(7) \\
\hline $\mathrm{O}(1)-\mathrm{C}(1)-\mathrm{C}(2)$ & $109.5(2)$ & $C\left(13^{\prime}\right)-C(14)-C(15)$ & $160.5(8)$ \\
\hline $\mathrm{O}(2)-\mathrm{C}(2)-\mathrm{C}(1)$ & $108.2(2)$ & $\mathrm{C}(13)-\mathrm{C}(14)-\mathrm{C}(15)$ & $115.0(7)$ \\
\hline $\mathrm{O}(2)-\mathrm{C}(3)-\mathrm{C}(4)$ & $108.9(3)$ & $C\left(14^{\prime}\right)-C(14)-C(16)$ & $154.2(9)$ \\
\hline $\mathrm{O}(3)-\mathrm{C}(4)-\mathrm{C}(3)$ & $108.8(3)$ & $\mathrm{C}\left(13^{\prime}\right)-\mathrm{C}(14)-\mathrm{C}(16)$ & $67.1(6)$ \\
\hline $\mathrm{O}(3)-\mathrm{C}(5)-\mathrm{C}(6)$ & $108.8(3)$ & $\mathrm{C}(13)-\mathrm{C}(14)-\mathrm{C}(16)$ & $112.6(7)$ \\
\hline $\mathrm{O}(4)-\mathrm{C}(6)-\mathrm{C}(5)$ & $108.9(3)$ & $C(15)-C(14)-C(16)$ & $132.4(6)$ \\
\hline $\mathrm{O}(4)-\mathrm{C}(7)-\mathrm{C}(8)$ & $108.8(3)$ & $C(13)-C\left(14^{\prime}\right)-C(14)$ & $92.6(8)$ \\
\hline $\mathrm{O}(5)-\mathrm{C}(8)-\mathrm{C}(7)$ & $108.3(3)$ & $\mathrm{C}(13)-\mathrm{C}\left(14^{\prime}\right)-\mathrm{C}\left(13^{\prime}\right)$ & $43.9(6)$ \\
\hline $\mathrm{C}(14)-\mathrm{C}\left(14^{\prime}\right)-\mathrm{C}\left(13^{\prime}\right)$ & $48.8(5)$ & $\mathrm{C}\left(13^{\prime}\right)-\mathrm{C}\left(14^{\prime}\right)-\mathrm{C}(18)$ & $116.2(6)$ \\
\hline$C(13)-C\left(14^{\prime}\right)-C(18)$ & $72.3(8)$ & $C(13)-C\left(14^{\prime}\right)-C(15)$ & $159.6(9)$ \\
\hline
\end{tabular}




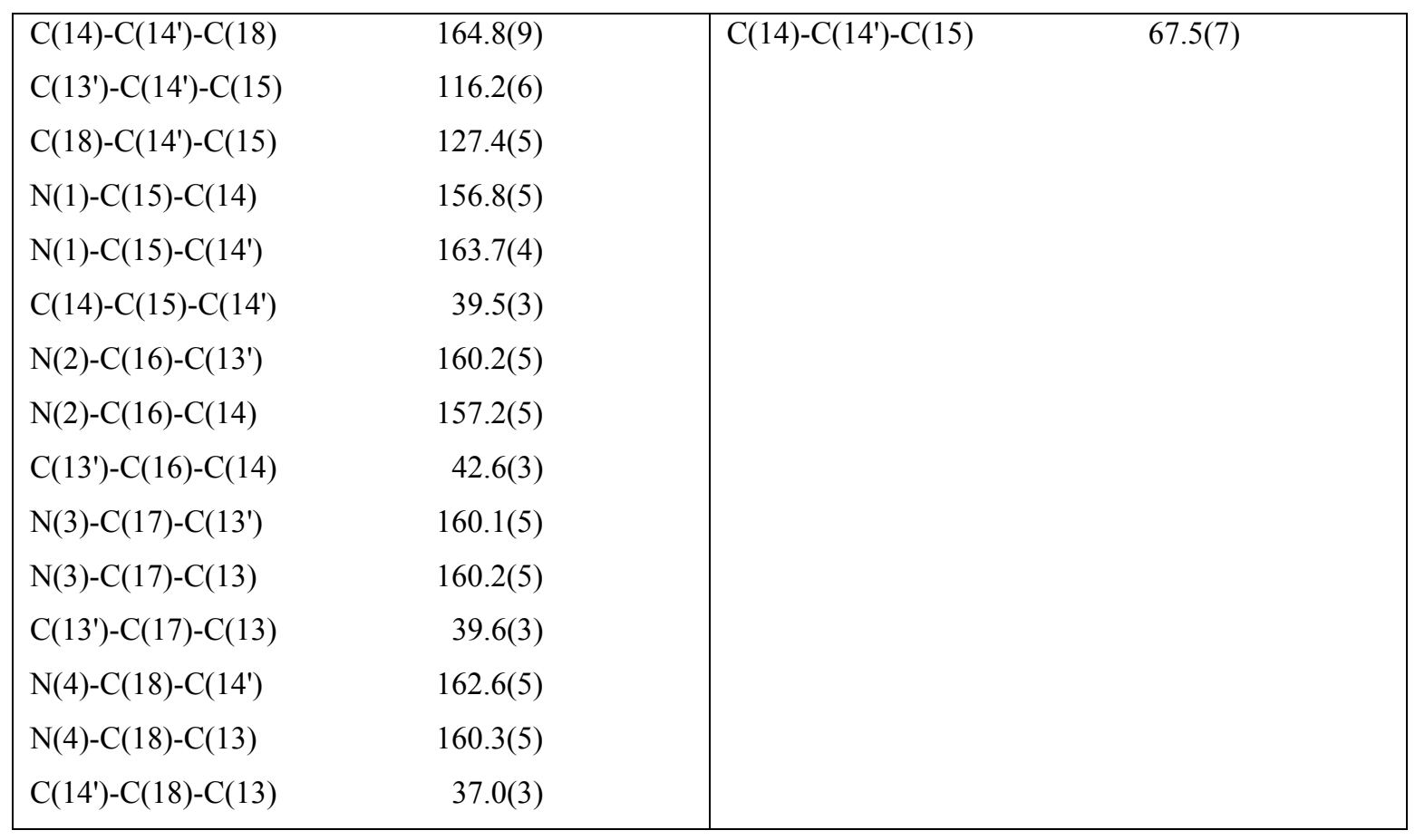

